\title{
Survive for split tooth by intracanal adhesive with intentional replantation.
}

\author{
Masaru Igarashi ${ }^{1 *}$, Kayoko Kitajima ${ }^{2}$, Kyoko Arai $^{2}$, Tomonori Satoh ${ }^{2}$ \\ ${ }^{1}$ Department of Endodontics, The Nippon Dental University, School of Life Dentistry at Tokyo, Japan \\ ${ }^{2}$ Department of Endodontics, The Nippon Dental University, School of Life Dentistry at Niigata, Japan
}

\begin{abstract}
The purpose of this case report was to describe the procedures and prognosis of intracanal adhesive treatment and intentional replantation for maxillary molar with crown/root fracture. The case of a vital tooth with buccal and lingual fragments caused by crown/root fracture was presented. The involved tooth was extracted and stored in balanced salt solution. Following confirmation of the repositioning of two fragments, the fractured surfaces of fragments were cut with a diamond bar until the cementum side remained. Finally, a large cavity was prepared in pulp chamber and the root canals like shell form. For the purpose of reinforcement after adhesion, a $U$-shaped orthodontic wire was bended which straddles the palatal canal and buccal canal. After drying the cavity wall, the dentin surface was treated with dentin conditioner, and the dual curing composite resin and the wire were filled in the cavity. After apicoectomy and retro filling, the tooth was replanted to original tooth socket. The prognosis was preferable at 1 month later and the tooth was used for an abutment of fixed metal bridge. The tooth still works in the mouth at the 3-year follow-up. Our results showed that intentional replantation after internal adhesive treatment with dual-cure resin and a metal wire is an effective and practical treatment for crown/root fracture of the maxillary molar.
\end{abstract}

Keywords: Crown/Root fracture, Intracanal adhesion, Intentional replantation, Dual-cure resin, Microscope.

Accepted on October 1 ${ }^{\text {st }}, 2019$

\section{Introduction}

Crown/root fracture is one of vertical root fractures (VRF) and the fracture line reaches from occlusal surface to root beyond the anatomical cervical line. VRF causes endodonticperiodontal disease of the tooth and is the worst disorder to which tooth extraction is indicated [1]. In recent years, improvement of the adhesive dental material has progressed, and a system which strongly adheres to dentin is used for VRF treatment [2-10]. Among them, the dual cure type core resin is a superior material with strong adhesiveness having light and chemical curability $[11,12]$. In addition, 4 meta-based resins are also used for attachment of prostheses, adhesion of root canal filling material and tooth fracture tooth, from adhesiveness and biocompatibility. In recent years, many clinicians have reported a technique to adhere fracture fragments, but because there is a thin material on the adhesion surface, there is anxiety about resistance to repetitive occlusion functional pressure applied postoperatively. Therefore, when adhesion healthy dentin surfaces, it was thought that increasing the thickness of the adhesive material on the fracture surface can get increase the mechanical strength $[13,14]$. In this method, intentional replantation is used in combination, and adhesion surfaces are formed in a hand-held situation, and a core resin is fill to the completely dried root canal surface $[15,16]$. In this report, the procedures and prognosis of the new treatment for the vertical tooth root fracture tooth reconstructed by intracanal adhesion method by intentional replantation.

\section{Material and Methods}

The procedures of intracanal adhesive and intentional replantation were described as follows.

- Local anesthesia: 2\% lidocaine with 1/80,000 adrenalin.

- Extraction with dental extracting forceps.

- Storing in cold saline.

- Removal of debris on the fractured surfaces.

- Confirmation replacement of separated fragments.

- Covering the root surface with wet gauze containing PBS and plastic lap.

- If need the root canal treatment, root canals should be shaped and obturated immediately.

- Making a big root canal cavity by cutting the fractured surface of each fragments with a diamond bar until the cementum side remained. (Save dentin just below the cement as thin as possible under the microscope observation.)

- Final preparation of a large and smooth cavity under repositioning the fragments. 
- Bending a U-shaped $0.9 \mathrm{~mm}$ wire across each root canal and check fit.

- Drying the root canal cavity and treat the root canal wall with dentin premier.

- Filling by injection the dual curing composite core resin into the cavity.

- Embedding the wire in the resin and irradiate the light.

- Cutting the $3 \mathrm{~mm}$ of root apex and prepare a retrofilling cavity.

- Filling MTA cement, EBA or composite resin in the cavity.

- Replantation the adhered tooth to the original tooth cavity.

- Suturing the tooth with a suture crossing the occlusal face.

- Removal of sutures after 7 days.

- Starting the prosthetic device 1 month later.

\section{Case Study}

A 51-year old man, the patient visited our university clinic for treatment of a crown root fracture of the maxillary second molar one month after the fracture (Figure 1). The fracture happened when the referral doctor was going removal of the metal inlay.

The involved tooth was separated into two sides and had a fracture line that runs in the mesio-distal position on the occlusal surface. Both separated pieces showed a $1 \mathrm{~mm}$ mobility by palpation with dental explorer horizontally (Figure 2). The tooth was diagnosed vertical crown root fracture as impossible to conserve and tooth extraction was confirmed.

Since there was little damage to the periodontal ligament of the broken fragments and the two pieces were completely able to replace, the method of replantation after applying the intracanal adhesion with composite resin was selected.

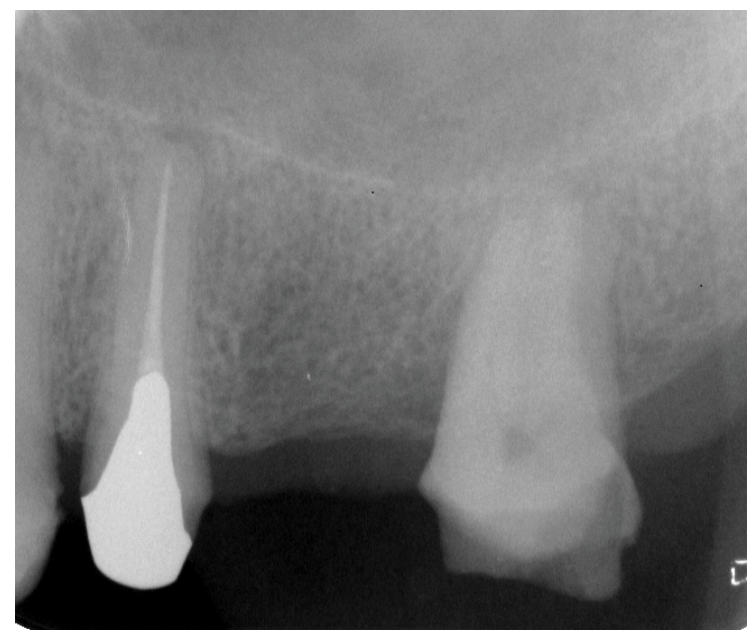

Figure 1. X-Ray of the upper second molar at the first visit.
The root of a fractured fragment was covered with wet gauze containing PBS. The root canal treatment was started, and the access was opened with tapered diamond bar (Figures 3 and 4). The root canal was shaped with NiTi rotary file (WaveOne Gold, Dentsply Sirona, Switzerland, Figure 5), and obturated with warm gutta-percha point and sealer (Thermafill and AH, Dentsply Sirona, Switzerland, Figure 6). The fractured surface of the tooth was cut with diamond bar under dental microscope viewing, until the intact dentin just below the cementum was saved as thin as possible (Figure 7). The same treatment was done for the other separated fragment.

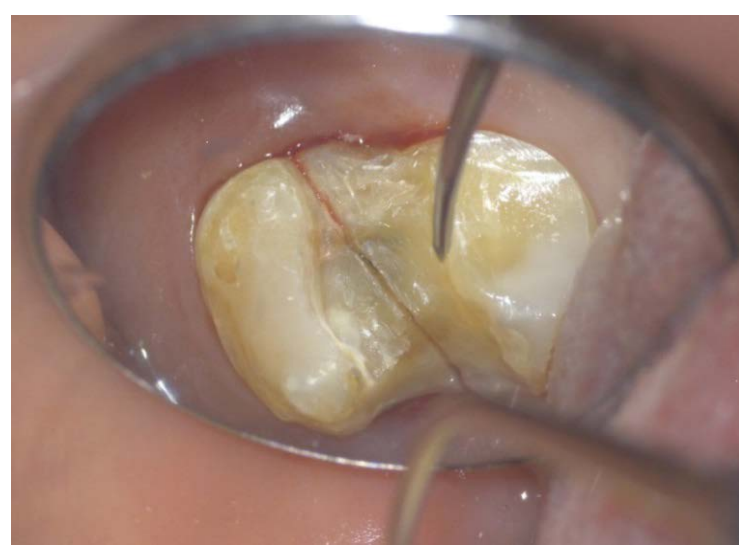

Figure 2. Oral view of the mesio-distal fracture line.

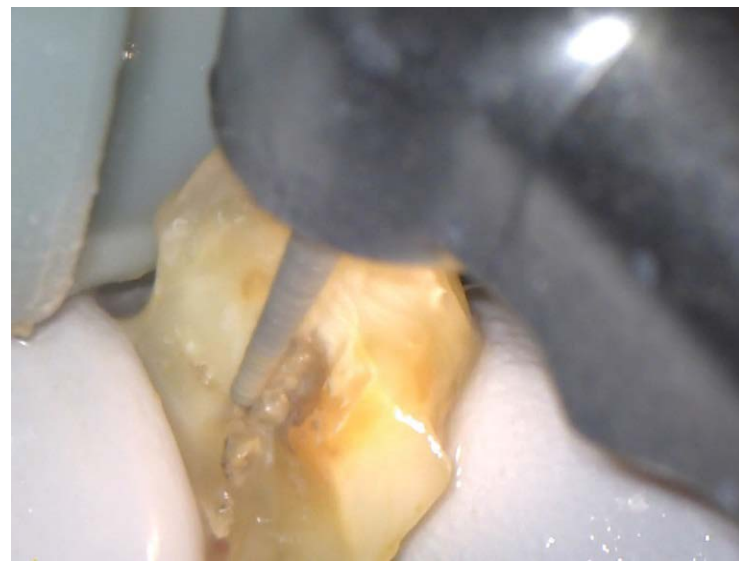

Figure 3. Access opening with tapered diamond point.

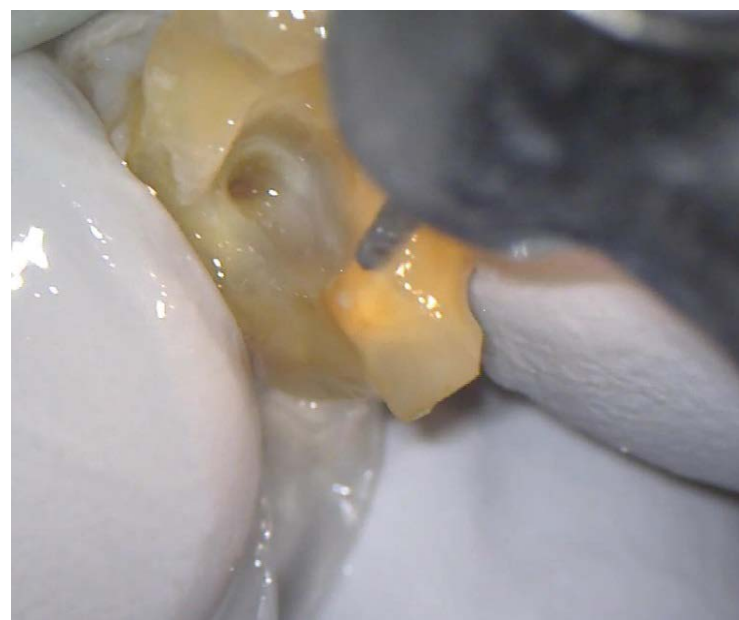

Figure 4. Enlargement of pulp chamber. 


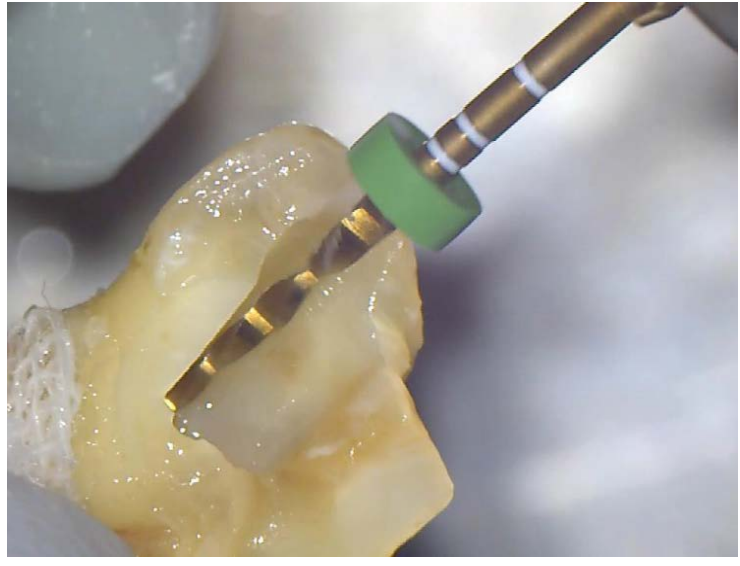

Figure 5. Root canal preparation by NiTi rotary file.

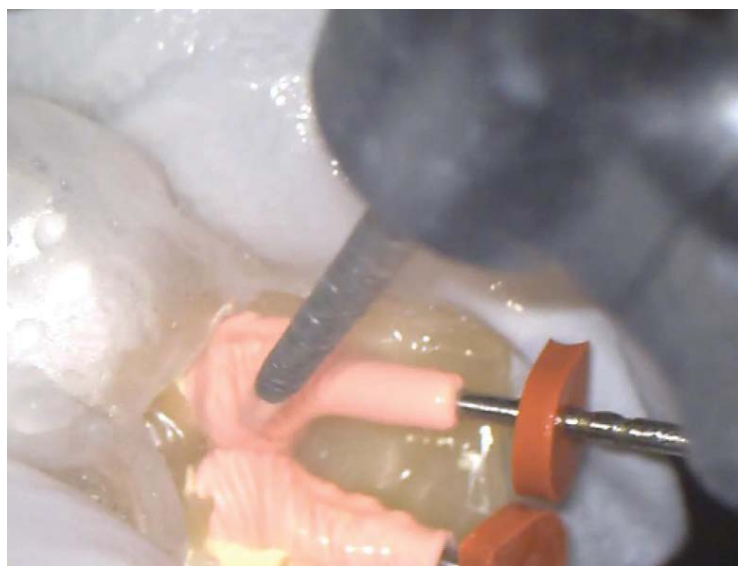

Figure 6. Root canal filling with hot gutta-percha.

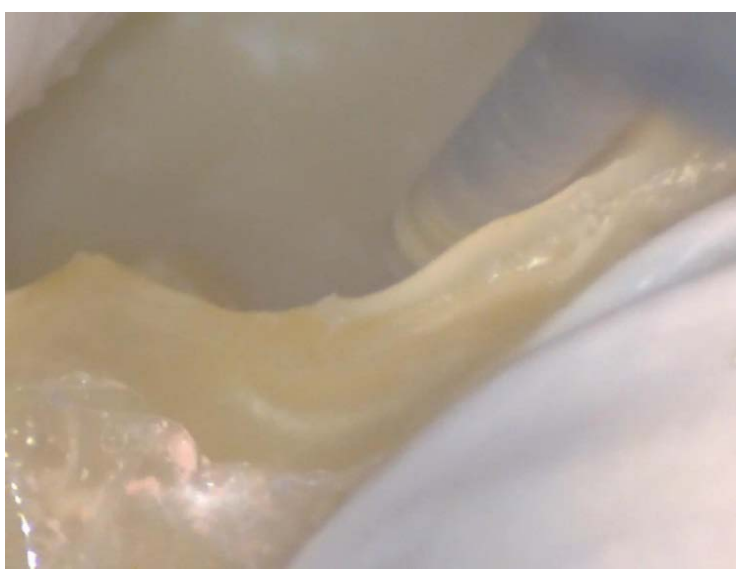

Figure 7. Removal of the fractured dentin surface just below the cementum.

The both fragments had been repositioned each other and confirmed the fitness (Figure 8). Then the post cavities of the root canals were prepared at each root canals. A U-shaped 0.9 $\mathrm{mm}$ stainless steel wires was prepared and checked the fitness to the post cavity (Figure 9). The whole root canal cavity was dried with a small suction tube and apply adhesive dentin primer to dentin. The dual cure composite core resin material (Clearfill DC core Auto mix ONE, Kuraray Noritake Dental) was injected into the root canal cavity and the U-shaped wire was embedded in the cavity and curing light was irradiated
(Figure 10). Finally, the adhesive fractured tooth was replanted to the original tooth cavity and fixed gently with a crossed simple suture (Figure 11).

One week later the suture was removed. There was no tooth mobility. The prosthodontic treatment was started one month later and the metal bridge was set. The fair progress was obtained even after 3 years (Figure 12).

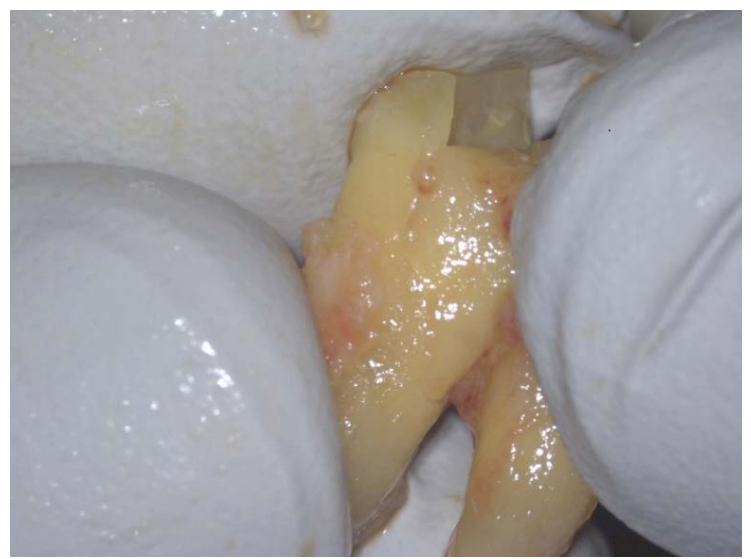

Figure 8. Confirmation of replacement of fragments.

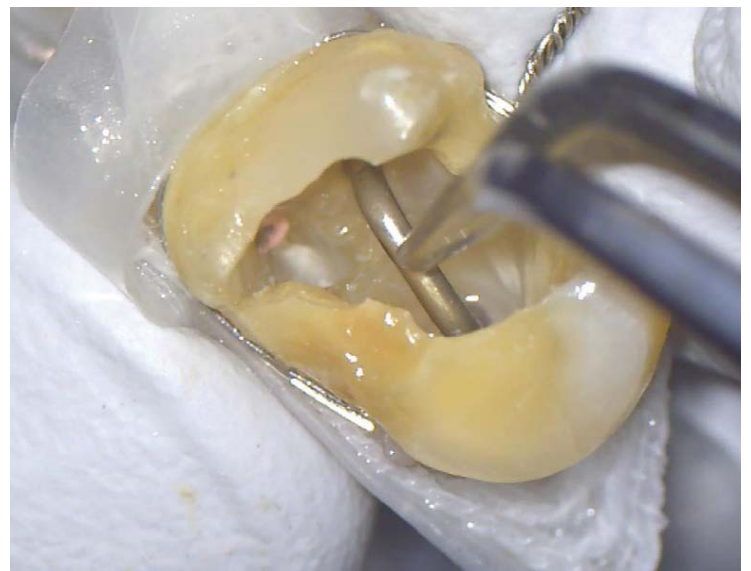

Figure 9. Fitting test of U-shaped wire.

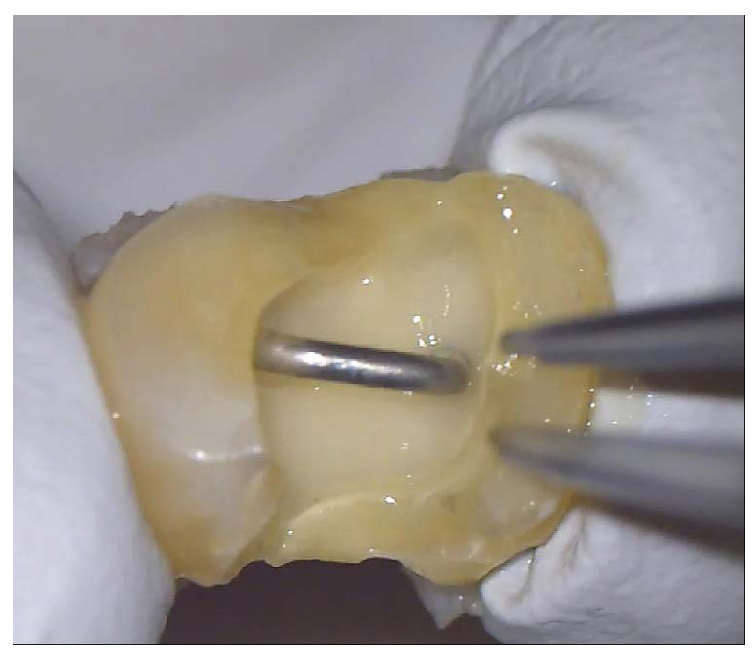

Figure 10. Internal adhesion with dual cure core resin with wire. 


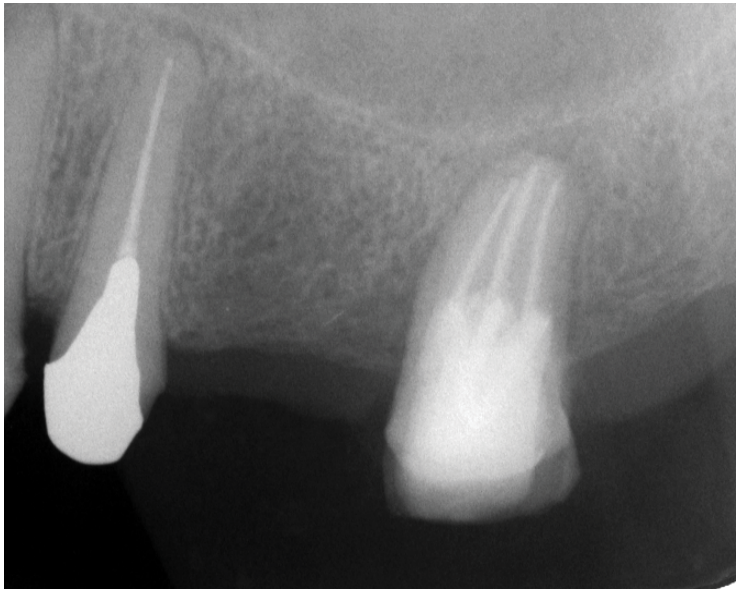

Figure 11. X-Ray at 1week after intentional replantation.

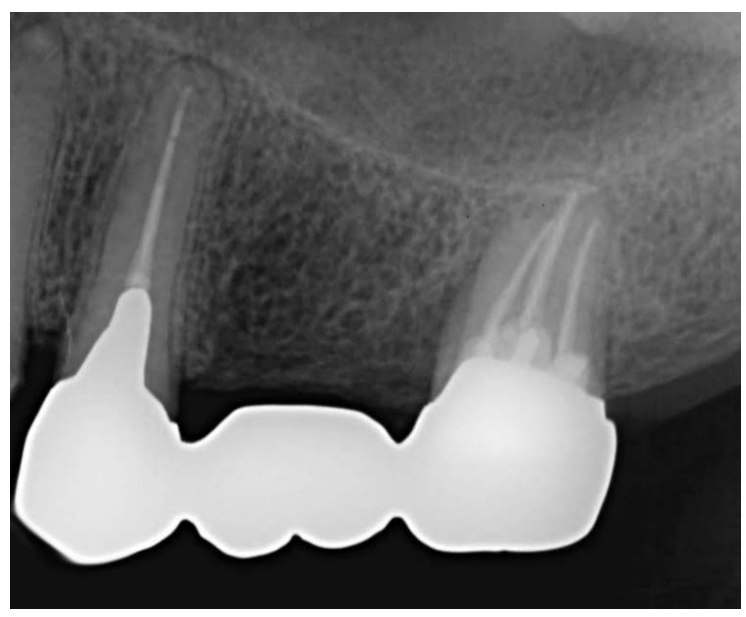

Figure 12. Postoperative X-Ray after $3 y$ after intentional replantation.

\section{Discussions and Conclusions}

In restorative dentistry, the improvement of adhesion method using composite resin has been advanced and strength bonding can be expected [11]. As a method of adhering to a split tooth, a method of cutting the fracture surface further and returning the tooth to its original shape with an adhesive material has been reported [16]. As a method of adhering to a split tooth, a method of cutting the fracture surface further and returning the tooth to its original shape with an adhesive material has been reported. In addition, while observing the teeth in the oral cavity with a microscope, a new procedure has also been reported in which the tooth material is cut along the fracture line from the inside of the root canal and removed to the vicinity of the root side of the root to adhere. In that case, since the adhesive material is applied to the healthy dentin surface after cavity preparation, the adhesive effect is increased more than the operation method of flowing the adhesive material into the root canal [13]. However, exudates or bleeding often occur in the root canal from the broken line during the adhesion work performed in the oral cavity, and it is difficult to perform the adhesion operation in a completely dry state. Therefore, in this method, a method of adhering tooth fracture by intentional replantation was selected. Since carried out by hand, it is possible to remove the dentin on the fracture surface to the vicinity of the root surface, so it is possible to increase the adhesion area. Therefore, as the thickness of the adhesive material increases, it can be expected that mechanical tectonic will also be larger than the film-like shape. In addition, because the dry state can be maintained, no exudates are generated in the root canal cavity, so that a reliable bonding operation can be performed.

In the cavity of the fracture surface, there is a large amount of food debris, bacteria, necrotic substances, flame-causing substances such as exudate denatured substances, which removal guarantees healing. Mechanical removal is the most convenient method for debridement and treatment under microscope can increase certainty. Even in this procedure, it was possible to remove the fracture surface from the root canal toward the root surface while observing with the microscope and to cut and remove as much as possible to the vicinity of the cementitious material. Even if all the fragments of dentin are removed to the vicinity of the cementitious material, when they are repositioned, the tooth root appearance is completely intact and only a large cavity is formed in the root canal. If the fragments of fractured tooth cannot be restored to original shape due to complex root fractures or the lack of some pieces, the treatment will be impossible. Furthermore, it is possible to change the fracture tooth to a crack tooth on the root surface. By filling the cavity with the composite resin for the core inside its root canal, it becomes a composite resin tooth having mechanical properties similar to teeth. That is, the ratio of the content of the core resin to the teeth becomes high, and the dentin is reborn as a composite resin tooth having cementum and periodontal ligament. A crack is an event seen in many teeth, and if the patient enforces plaque control it will not cause great damage. Therefore, it was possible to revive the teeth of the remaining life 0 day which became tooth extraction by making perfect squirrel teeth as crack teeth. Although it is possible that pocket formation may remain along the crack, it is thought that the infection control mechanism of the living body can be fully demonstrated by thorough plaque control of the cracked part. Biological healing is expected as the patient's own teeth are replanted. Since the reconstructed tooth has healthy dentin and cementum and periodontal ligament tissue around the core resin, it can have the same healing process as normal teeth. Moreover, since most of the broken teeth are composite resins, the strength of the teeth increases.

In recent years, tooth reinforcement has been done in fiber posts [13], but fiber posts cannot be used dental alone as a root canal, it is impossible to cross the root canal. Therefore, a method of reinforcing by inserting a straightening 0.9 $\mathrm{mm}$ wire from the root canal opening into the root canal was attempted, but it is easy to bend in accordance with the opening of the root canal of the tooth, and the insertion depth is also a length [16]. It was a reliable method because it can only be adjusted. This method is a revolutionary method applicable also to single root teeth such as tongue fracture 
of the maxillary bicuspid, mandibular molar and front teeth. Even in this case, since it functions as a bridge abutment for the first molar defect, it can be said that it is a new treatment method in the future. On the other hand, intentional replantation is concerned about external absorption of the anchyloses after surgery, but since the dental practitioner can treat it under the preservative environment and under the protection of the periodontal membrane, there are not many such discomfort prognosis. Also, in implants with artificial materials, percutaneous inflammation after operation is likely to occur due to absent of the periodontal ligament tissue, but defense mechanism works on your teeth. This method can be said to save the broken tooth in cracked tooth. This method can also be considered to make a resin tooth with a periodontal ligament that makes up many of the resin for the core using patient's own teeth. In future the more replanted cases will research the prognosis and improve the surgical procedure.

Vertical root fractured tooth is able to be survived by using adhesive composite resin in the root canals with application of the intracanal adhesive resin under the intentional replantation.

\section{Conflict of Interest}

The authors declare that they have no competing interests. Informed consent about this presentation has been obtained and the ethics number is ECNG-H-41(The Committee of the School of Life Dentistry at Niigata, The Nippon Dental University, Niigata, Japan).

\section{References}

1. Polson AM. Periodontal destruction associated with vertical root fractures. J Periodontol. 1977;48:27-32.

2. Sugaya T, Kawanami M, Noguchi H, et al. Periodontal healing after bonding treatment of vertical root fracture. Dent Traumatol. 2001;17:174-9.

3. Özer SY, Ünlü G, Değer Y. Diagnosis and treatment of endodontically treated teeth with vertical root fracture: Three case reports with two-year follow-up. J Endod. 2011;37(1):97-102.

4. Hayashi M, Kinomoto Y, Miura M, et al. Short-term evaluation of intentional replantation of vertically fractured roots reconstructed with dentin-bonded resin. J Endod. 2002;28:12-4.

5. Kawai K, Masaka N. Vertical root fracture treated by bonding fragments and rotational replantation. Dent Traumatol. 2002;18:42-5.

6. Kudou Y, Kubota M. Replantation with intentional rotation of a complete vertically fractured root using adhesive resin cement. Dent Traumatol. 2003;19:115-7.

7. Hayashi M, Kinomoto Y, Takeshige F, et al. Prognosis of intentional replantation of vertically fractured roots reconstructed with dentin-bonded resin. J Endod. 2004;30:145-8.

8. Oztürk M, Unal GC. A successful treatment of vertical root fracture: a case report and 4-year follow-up. Dent Traumatol. 2008;24(5):e56-60.

9. Arikan F, Franko M, Gurkan A. Replantation of a vertically fractured maxillary central incisor after repair with adhesive resin. Int Endod J. 2008;41(2):173-9.

10. Deepti Dua, Ankur Dua. Reconstruction and intentional replantation of a maxillary central incisor with a complete vertical root fracture: A rare case report with three years follow up. J Clin Diagn Res. 2015;9:6-9.

11. Nishimura Y, Tsubota Y, Yamada Y, et al. A study on bond strength of prefabricated metal posts to composite resin core material prefabricated metal post, composite resin core material, shear bond strength, adhesion: Adhesive dent. 2002;20(2):5967.

12. Moradi Majd N, Akhtari F, Araghi S, et al. Treatment of a vertical root fracture using dual-curing resin cement: a case report, Case Rep Dent. 2012;985215.

13. Amakawa A, Tani-Ishii N. The clinical effects of internal rootdentin adhesion therapy for vertical root fracture. J Japan Endod. 2010;31(30):182-7.

14. Cho SY, Lee Y, Shin SJ, et al. Retention and Healing Outcomes after Intentional Replantation. J Endod. 2016;42(6):909-15.

15. Okaguchi M, Kuo T, Ho YC. Successful treatment of vertical root fracture through intentional replantation and root fragment bonding with 4-META/MMA-TBB resin. J Formos Med Assoc. 2019;118(3):671-8.

16. Igarashi M, Kitajima K, Arai K. Application of internal adhesive therapy and intentional replantation for maxillary premolars with crown/root fracture. J Japan Endod Assoc. 2017;38(2):114-121.

\section{*Correspondence to:}

Masaru Igarashi

Department of Endodontics

School of Life Dentistry at Tokyo

The Nippon Dental University

1-9-20 Fujimi, Chiyoda-ku, Tokyo 102-8159

Japan

Tel: +81-3-3261-5695

E-mail: m-igarashi@tky.ndu.ac.jp 